\title{
Alpha Particle-Driven Toroidal Alfvén Eigenmodes in Tokamak Fusion Test Reactor Deuterium-Tritium Plasmas: Theory and Experiments
}

\author{
G. Y. Fu, R, Nazikian, R. Budny, Z. Chang \\ Princeton Plasma Physics Laboratory, Princeton University \\ P. O. Box 451 \\ Princeton, New Jersey 08543, U.S.A.
}

\begin{abstract}
The toroidal Alfvén eigenmodes (TAE) in the Tokamak Test Fusion Reactor [K. Young et al., Plasma Phys. Controlled Fusion 26, 11 (1984)] DeuteriumTritium plasmas are analyzed using the NOVA-K code [C. Z. Cheng, Phys. Reports 211, 1 (1992)]. The theoretical results are compared with the expermental measurements in detail. In most cases, the theory agrees with the observations in term of mode frequency, mode structure and mode stability. However, one mode with toroidal mode number $n=2$ is observed to be poloidally localized on the high field side of the magnetic axis with a mode frequency substantially below the TAE frequency.

52.35.Bj, 52.65.+z, 52.55.Pi
\end{abstract}

Typeset using REVTEX 


\section{INTRODUCTION}

In a tokamak reactor with ignited Deuterium-Tritium (DT) plasma, the fusion product alpha particles are born with an energy of $3.5 \mathrm{MeV}$ and a pressure profile highly peaked at the center of the plasma. The energetic alpha particles can heat the plasma via collision with thermal electrons, but they can also drive collective instabilities with the free energy in the alpha particle pressure gradient. In particular, the Toroidal Alfvén Eigenmode can be destabilized by super-Alfvénic alpha particles via parallel wave particle resonances ${ }^{1}$. Such alpha-driven instability could cause significant alpha particle loss, serious damage in the reactor's first wall, and degrade alpha particle heating. Previously, the alpha particle destabilization of TAE was simulated experimentally in non-fusion plasmas using energetic beam ions from Neutral Beam Injection (NBI) heating ${ }^{2,3}$ or fast hydrogen minority tail ions powered by Ion Cyclotron Range of Frequency heating ${ }^{4-9}$. Strong TAE activity and associated fast ion loss were observed in these experiments.

Purely alpha-driven TAE instability was observed for the first time in the Tokamak Fusion Test Reactor $(\text { TFTR })^{10}$ DT plasmas with weak central shear and $q(0)>1^{11,12}$, where $q(0)$ is the value of the safety factor at the magnetic axis. The mode activity appears after termination of NBI, as predicted by theory ${ }^{13,14}$. Furthermore, the mode structure has been obtained with the core reflectometer diagnostic ${ }^{15}$. It was observed that the instability with $n>3$ is core-localized and exhibits ballooning feature, i.e., the mode is poloidally localized on the low field side of the magnetic axis on the plasma midplane (Here $n$ denotes the toroidal mode number). These results confirm the theoretical predication of mode localization ${ }^{14}$ and the importance of high $q(0)$ for mode excitation ${ }^{16}$. However, the $n=2$ mode is localized on the high field side with a mode frequency inside the continuum spectrum. Both the low mode frequency and spatial structure contradicts the usual expectation of TAE modes.

In the present work, we report on a detailed and systematic comparison between theory 
and experimental measurements. The physics aspect of the alpha-driven TAE is emphasized in the comparison. This paper is complementary to the work of Nazikian et $a l .{ }^{15}$ where detailed experimental data of alpha-driven TAE in TFTR are presented along with initial comparison with theory.

The paper is organized as follows. In Sec. II, essential physics of this work is discussed. In Sec. III, the experimental observations are briefly described. Section IV presents detailed comparison between theory and experiment. Finally, discussion and conclusions are given in Sec. V.

\section{THEORETICAL BACKGROUND}

The TAE modes are discrete shear Alfvén waves with frequencies located inside the continuum gaps in a toroidal plasma ${ }^{17}$. In a cylindrical plasma, the shear Alfvén spectrum is given by $\omega^{2}=k_{\|}^{2}(r) v_{A}^{2}(r)$, where $v_{A}=B / \sqrt{\rho}$ is the Alfvén speed and $k_{\|}=(n-m / q) / R$ is the parallel wave number with $B$ being the magnetic field strength, $\rho_{m}$ the mass density, $m / n$ the poloidal/toroidal mode number, and $q$ the safety factor. The cylindrical continua are degenerate at $q=(2 m+1) / 2 n$ where $k_{\|, m}^{2}=k_{\|, m+1}^{2}$ and the mode frequency of harmonic $m$ equals that of $m+1$. In a torus, however, the frequency degeneracy is eliminated by the poloidal asymmetry (e.g., $B$ varies in poloidal angle due to toroidicity.) . The toroidal coupling of $m$ and $m+1$ creates a gap in the continuum spectrum at the radius of $q=$ $(2 m+1) / 2 n^{18}$. More interestingly, discrete eigenmodes have been shown to exist inside the continuum gap ${ }^{17}$. These modes are called Toroidicity-induced shear Alfvén Eigenmode $(\mathrm{TAE})^{1}$ or Toroidal Alfvén Eigenmode.

In the original work of $\mathrm{TAE}^{17}$, only one TAE mode was shown to exist in a single continuum gap at zero beta. At finite plasma beta, the TAE mode can exist only below a critical pressure gradient slightly below the first ballooning stability boundary ${ }^{19}$. Near the center of plasma, the magnetic shear tends to be small, and the TAE mode is core- 
localized $^{20}$ at a single gap with only two appreciable poloidal components. The critical gradient is given ${ }^{21}$ by $\alpha=2 s^{2}+3 \epsilon$ where $\alpha=-R q^{2}(d p / d r) / B^{2}$ is the normalized pressure gradient, $s$ the magnetic shear, $\epsilon=r / R$ the inverse aspect ratio. In addition to the usual TAE (even parity with frequency near the lower bound of a continuum gap), it has been shown $^{22}$ that another TAE can also exist in the core of plasma with frequency slightly below the upper bound of the gap. This mode is called odd TAE because the phases of the two dominant poloidal harmonics are opposite. The corresponding critical pressure gradient is given by $\alpha=3 \epsilon-2 s^{2}$. These core-localized modes can exist at finite beta because of finite aspect ratio effects which were neglected in previous studies. The analytic results are valid when $s^{2} \sim \epsilon<<1$. More recently, It has been shown ${ }^{23}$ that more than two TAE can exist in a single gap when $\epsilon / s \sim O(1)$.

Besides core-localized modes, global TAE modes also exist with multiple poloidal harmonics radially spanning multiple continuum gaps. ${ }^{14}$

When kinetic effects of thermal electrons and ions, such as Finite Ion Larmor Radius (FLR) and finite electron mass, are taken into account, the continuum spectrum is discretized. In particular, the Kinetic Toroidal Alfvén Eigenmode (KTAE) can exist with frequency just above the upper bound of the gap ${ }^{24}$. The KTAE is a radially standing wave formed due to the toroidal coupling of two kinetic Alfvén waves having poloidal mode number $m$ and $m+1$ respectively.

TAE/KTAE are two types of many Alfvén Eigenmodes. Other types include the Betainduced Alfvén Eigenmode (BAE) ${ }^{25}$, the Ellipticity-induced Alfvén Eigenmode (EAE) ${ }^{26}$, the Non-circularity triangularity-induced Alfvén Eigenmode (NAE) ${ }^{26}$, the Global Alfvén Eigenmodes ${ }^{27}$ (GAE) or the Discrete Alfvén Waves $^{28}$ (DAW), the Kinetic Alfvén Wave ${ }^{29}$ (KAW), the Energetic Particle-induced Alfvén Mode ${ }^{30}$ (EPM) or the Resonant Alfvén Eigenmode $^{31}$ (RTAE). However, TAE/KTAE are most susceptible to alpha particle destabilization for two reasons. First, the background damping of the mode is relatively low due to lack of continuum damping. Second, the mode frequency is relatively small so that the condition $\omega_{*, \alpha}>>\omega$ is well satisfied, where $\omega_{*, \alpha}$ is the alpha particle diamagnetic drift 
frequency.

The alpha-particle destabilization of shear Alfvén waves was first considered by Rosenbluth and Rutherford ${ }^{32}$, and Mikhalovaskii ${ }^{33}$. Since the alpha particle's speed is comparable to the Alfvén speed for typical fusion plasma parameters, they can resonantly interact with shear Alfvén waves $\left(\omega=k_{\|} v_{\|}\right)$. The instability drive comes from the free energy associated with the alpha particle pressure gradient. The instability mechanism can be understood from single particle motion in the presence of a shear Alfvén wave with frequency $\omega$. Using the drift-kinetic equation, the rate of change in the particle kinetic energy $E_{k}$ is $d E_{k} / d t \approx \mathbf{v}_{\mathbf{d}} \cdot \mathbf{E}$, and the rate of change in the toroidal angular momentum $P_{\phi}$ is given by $d P_{\phi} / d t=(n / \omega) d E_{k} / d t$, where $\mathbf{v}_{\mathbf{d}}$ is the drift velocity, $\mathbf{E}$ is the electric field of the Alfvén wave, and $P_{\phi}=e \Psi+M v_{\phi} R$ with $e$ the ion charge, $\Psi$ the radial variable of poloidal flux, $M$ the ion mass, $v_{\phi}$ the toroidal component of the particle velocity, and $R$ the major radius. Since $P_{\phi} \approx e \Psi$ for small orbit width, $P_{\phi}$ can be thought as a radial variable. Thus, the change in particle energy is proportional to the change in particle radial position. For $n / \omega<0$, a resonant particle loses energy when it moves out in radius, and gains energy when moves in. Alpha particles on the whole lose energy to the TAE because there are more particles at smaller radii.

For TAE excitation, alpha particles must overcome the sum of all background damping due to kinetic effects of thermal electrons and ions, such as electron/ion Landau damping, collisional damping, continuum damping, and radiative damping. For typical parameters of TFTR DT experiments, the main damping mechanisms are radiative damping and beam ion Landau damping. The thermal ion Landau damping and the collisional damping are negligible. The continuum damping is not important in most cases and is not considered in our model.

The term of radiative damping was coined by Mett and Mahajan ${ }^{24}$ who pictured the damping process as the TAE mode "radiating" away kinetic Alfvén waves. Physically, the damping is caused by dissipation of short wave length kinetic Alfvén waves which are coupled to the usual MHD TAE mode. In the ideal MHD limit, the TAE mode has a 
characteristic radial wavelength of $\lambda_{m} \sim \epsilon r / n s$. However, when FLR effects are taken into account, the TAE branch is coupled to the KAW branch with a short wave length on order of $\lambda_{k} \sim\left(\rho_{i}^{2} r / n q s\right)^{1 / 3}$ with $\rho_{i}$ being the ion gyroradius. This small wavelength component of FLR-modified TAE leads to finite damping caused by dissipations such as collision and eletron Landau damping. It should be pointed out that although non-zero dissipation is required for having finite radiative damping, the magnitude of the radiative damping is independent of dissipation when it is small. This is because the range of radial wave number $k_{r}$ of the TAE depends on the magnitude of the dissipation. The smaller the dissipation, the larger $k_{r}$. For this reason, the radiative damping is non-perturbative.

In the present work, the alpha drive and damping terms are calculated perturbatively using the kinetic-MHD stability code NOVA-K ${ }^{34,35,14}$. It should be noted that our perturbative method is valid for the parameters of TFTR DT experiments since the calculated drive and damping rates are smaller than the distance between the real frequency and the nearest edge of the continuum gap. The non-perturbative radiative damping is calculated semi-analytically using a WKB approximation ${ }^{14}$.

\section{EXPERIMENTAL OBSERVATIONS}

Purely alpha-particle-driven TAEs were observed in TFTR DT plasmas ${ }^{11,12,15}$. The TAE instability occurs only for weak central magnetic shear and $q(0)>1$ achieved using full radius plasmas during current ramp-up on TFTR. The modes, with toroidal mode number in the range of $n=2 \sim 5$, appear in a transient phase $100-300 \mathrm{~ms}$ following the end of DT neutral beam injection. Sometimes, $n=1$ and $n=6$ modes are also seen.

Figure 1 shows time traces of relevant plasma parameters for a typical DT plasma with $q(0) \sim 1.6$ (discharge \#103101) in which TAEs were observed. After the termination of NBI, the plasma density, alpha beta, as well as plasma beta decay in time. The mode activity is clearly seen $50-100 \mathrm{~ms}$ after the end of NBI as indicated in Fig. 2 by the amplitude of edge 

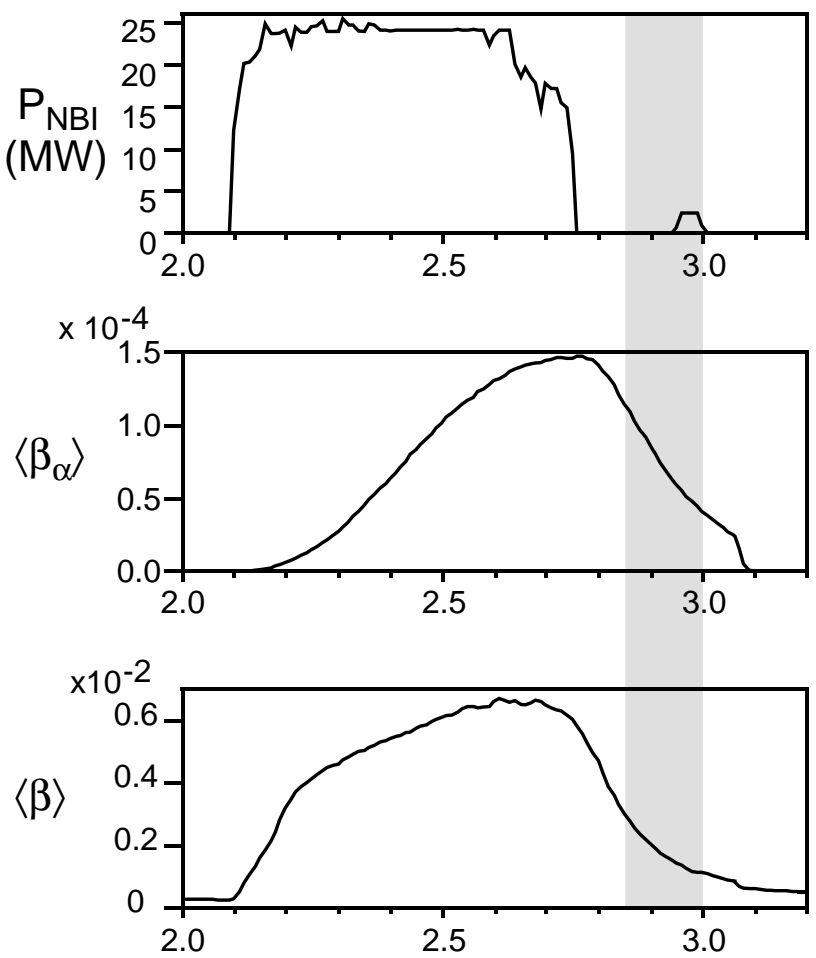

FIG. 1. Evolution of DT neutral beam power, volume-averaged alpha particle beta $\left\langle\beta_{\alpha}\right\rangle$, and volumed-averaged plasma beta $\langle\beta\rangle$ of a TFTR discharge (\#103101). The shaded region denotes time of TAE activity.

magnetic fluctuations and the density fluctuation measured by the reflectometer diagnostic. The modes have toroidal numbers ranging from $n=2-5$ and frequencies on order of $f \sim 150-250 \mathrm{kHz}$. No TAE activity was observed during the NBI heating. It should be pointed out that the alpha particle slowing-down time $(\sim 300-400 \mathrm{~ms})$ is sufficiently long so that the alpha particles can still resonate with TAE modes at the time of observed mode activity. In contrast, the plasma density and beta decay on a much short time scale $(\sim 100$ $\mathrm{ms})$.

The TAE activity is detected using both external and internal measurements. The edge magnetic fluctuations (poloidal magnetic field) associated with the modes are measured by the Mirnov coils which determines the mode amplitude evolution, mode frequencies and the toroidal mode numbers. The density fluctuation of TAEs is measured using the X-mode core 


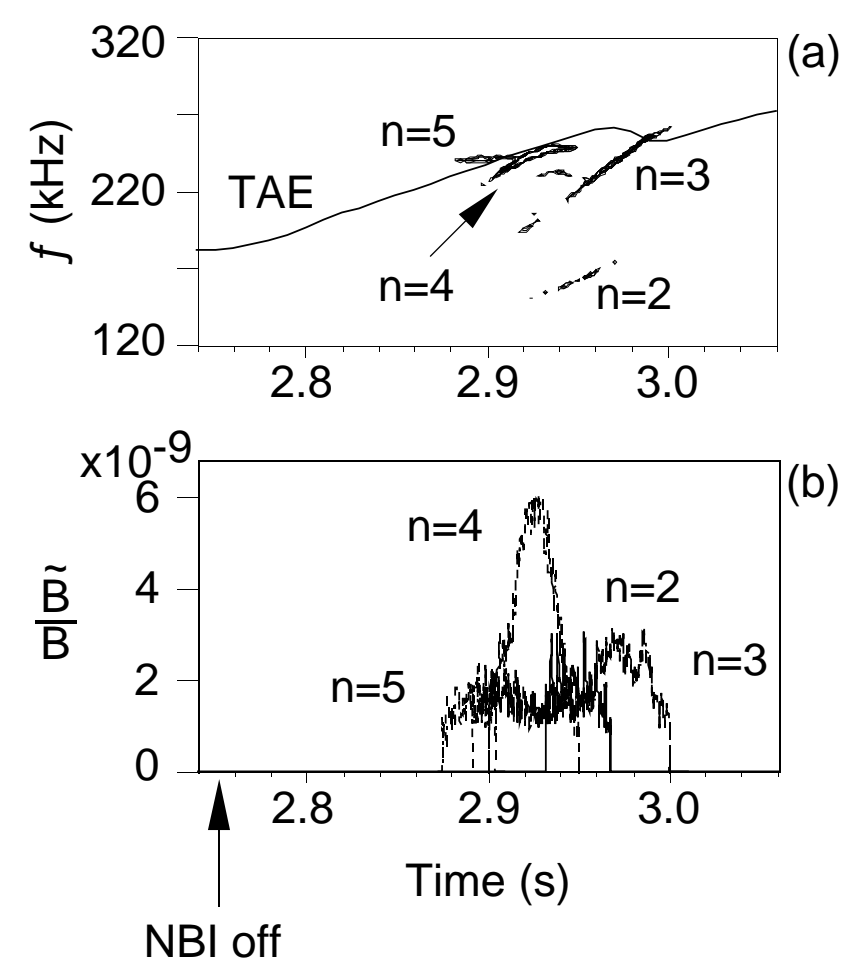

FIG. 2. Evolution of (a) frequency and (b) amplitude of edge magnetic fluctuations on outer midplane in the TFTR discharge (\#103101).

reflectometer diagnostic. This diagnostic measures phase fluctuations induced on a probing microwave beam reflected from density variations in the plasma. This measurement is nearly local becauses the phase fluctuations are mainly determined by the density variation at the cut-off layer of the $\mathrm{X}$-mode ${ }^{15}$. Because of the strong dependence of the cutoff position on the radially decreasing toroidal magnetic field strength, a radial scan of the mode structure can be obtained using the three fixed frequency channels (170 Ghz, $143 \mathrm{Ghz}$ and $135 \mathrm{Ghz}$ ) on TFTR by introducing a small (10\%) variation in the toroidal field strength keeping $q(a)$ constant. Figure 3 shows the radial structure of the phase fluctuations obtained from the radial scan of the cutoff layers using a succession of plasmas with reproducible mode activity as measured on external Mirnov coils. Two key results emerge from this figure. First, the modes are core localized at about $r / a=0.3$. Second, the $n=4$ mode exhibits ballooning mode structure (localized on low field side) as expected for the usual TAE whereas the $n=2$ mode has a strong anti-ballooning structure (i.e., the mode amplitude at the high field side 
is much larger than that on the low field side).
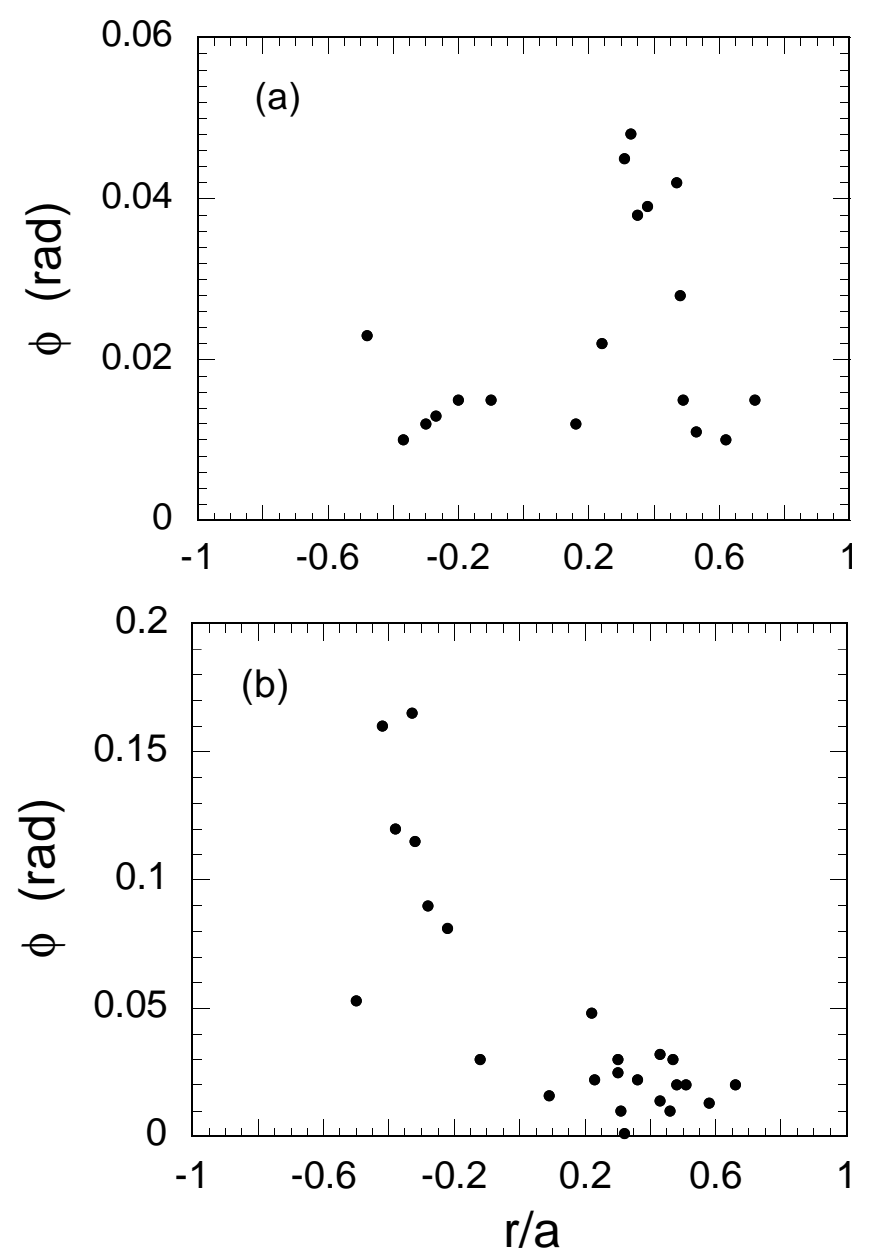

FIG. 3. Collection of the phase magnitude observed on the reflectometer for (a) $n=4$ mode and (b) $n=2$ mode taken over a range of similar plasmas with $\sim 10 \%$ variation in toroidal field keeping $q(a)$ constant. These plasmas showed nearly identical TAE activity. The data are taken at the peak of the edge magnetic signal for each mode.

The plasma parameters and profiles at the time of mode activity $(t=2.92 \mathrm{sec})$ are: the major radius $R=253 \mathrm{~cm}$, the minor radius $a=88 \mathrm{~cm}$, the toroidal magnetic field $B=5.08 \mathrm{~T}$, the plasma current $I_{p}=2.0 \mathrm{MA}$, the central plasma beta $\beta(0)=1.0 \%$, the volume averaged plasma beta $\langle\beta\rangle=0.19 \%$, the central alpha beta $\beta_{\alpha}(0)=0.06 \%$, the central electron density $n_{\epsilon}(0)=3.98 \times 10^{13} \mathrm{~cm}^{-3}$, the ion temperature $T_{i}(0)=11.3 \mathrm{keV}$, the electron temperature $T_{e}(0)=6.05 \mathrm{keV}$, The effective mass $A_{e f f}=2.04$, the effective charge $Z_{e f f}=2.44$. In the calculations, alpha particles are modeled with an isotropic slowing-down 
distribution. Figure 4 show the profiles of electron density, the safety factor $q$, the total plasma beta, the alpha beta, the ion and electron temperatures. These profiles are fitted analytically by

$$
\begin{gathered}
n_{e}=n_{e}(0)\left(1-1.39 \psi+1.44 \psi^{2}-1.64 \psi^{3}+1.72 \psi^{4}-0.94 \psi^{5}\right), \\
\beta=\beta(0)(1-\Psi)\left(1-2.20 \Psi+1.43 \Psi^{2}\right), \\
\beta_{\alpha}=\beta_{\alpha}(0)\left(1-\Psi^{1.23}\right)^{6.5},
\end{gathered}
$$

and

$$
q=q_{0}+\Psi\left[q_{1}-q_{0}+\left(q_{1}^{\prime}-q_{1}+q_{0}\right) \frac{\left(1-\Psi_{s}\right)(\Psi-1)}{\Psi-\Psi_{s}}\right]
$$

where $\Psi_{s}=\left(q_{1}^{\prime}-q_{1}+q_{0}\right) /\left(q_{0}^{\prime}+q_{1}^{\prime}-2 q_{1}+2 q_{0}\right), \Psi$ is the poloidal flux normalized to unity at the plasma edge and is zero at the magnetic axis, $q_{0}=1.59, q_{1}=4.93, q_{0}^{\prime}=0.42$ and $q_{1}^{\prime}=12.5$. The $q$ profile is measured using the MSE diagnostic.

These parameters and profiles are obtained from TRANSP ${ }^{36}$ simulations which are based on the experimental measurements of plasma density, temperature, and safety factor etc. In particular, the TRANSP code calculates the alpha distribution from the measured neutron rate and profile, using a Monte Carlo analysis with collisional slowing-down.

\section{COMPARISON BETWEEN THEORY AND EXPERIMENTS}

An essential feature of TAEs is that the mode frequency lies inside the toroidicityinduced shear Alfvén continuum gaps. Figure 5 shows the continuum spectrum and the corresponding measured mode frequency for $n=2,3$ and 4 modes respectively. We observe that the experimental mode frequencies for $n=3$ and $n=4$ are inside the continuum gaps throughout the entire plasma. However, the $n=2$ mode frequency is inside the continuum near the center of the plasma, although the frequency does lie inside the gaps near the edge of the plasma. Assuming the mode structures are core-localized at about $r / a=0.3$ 

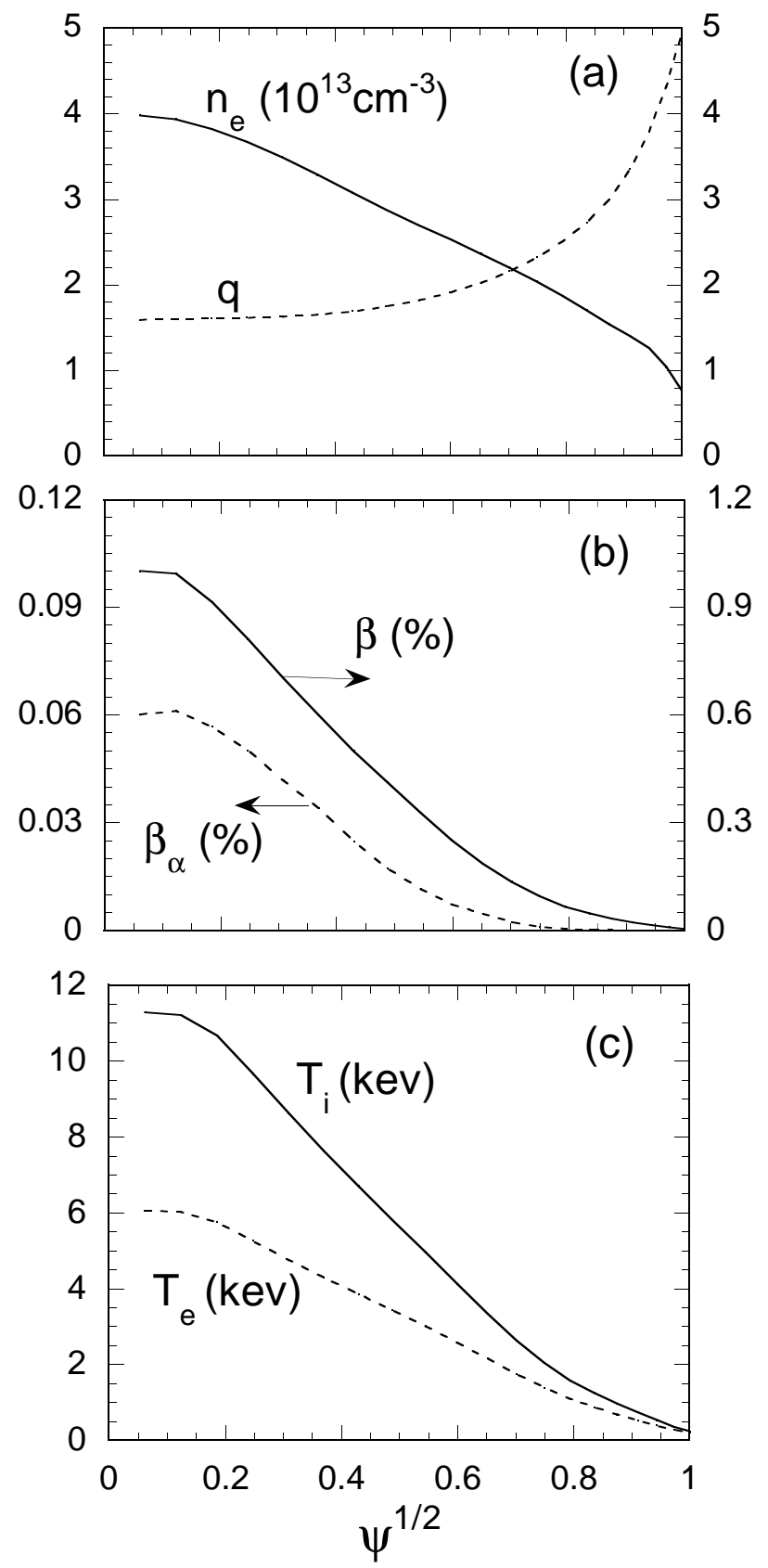

FIG. 4. The plasma profiles at $t=2.92 s e c$ in the DT discharge \#103101: (a) electron density $n_{e}$ and safety factor $q ;(b)$ plasma beta $\beta$ and alpha particle beta $\beta_{\alpha}$; (c)electron and ion temperatures. Here $\Psi$ is the poloidal flux normalized to unity at the edge and zero at the center. 
as observed in the experiments, then the mode frequencies for $n=3$ and $n=4$ are indeed consistent with TAE's, but the $n=2$ frequency is significantly lower than the theoretical expectation.

A more precise comparison is given in Fig. 6 where the calculated even core-localized TAE frequencies are compared with the measured values for $n=2-5$. It is clear that the experimental frequencies exhibit a much stronger dependence on mode number as compared to that of the calculated core TAE frequency. It is unclear how to resolve this discrepancy. Frequency correction due to kinetic effects or plasma rotation are much too small to account for the difference. For example, the plasma rotation is about $0.02 \mathrm{kHz}$ and the diamagnetic drift frequency is $7.4 \mathrm{kHz}$ at $r / a \sim 0.3$ and $t=2.92 \mathrm{sec}$. The alpha particle effect on the real part of the mode frequency is also expected to be small. The frequency correction due to the alpha particle kinetic effect is estimated to be about $1 \%$ of the mode frequency.

In addition to the even core-localized mode, the odd core-localized modes and global modes can also exist in the continuum gaps. For $n=4$, there are a total of five modes whose radial eigenmode structures are shown in Fig. 7. The corresponding perturbed densities are shown in Fig. 8. Table I lists the mode frequency, mode type, and ratio of alpha drive and damping for each of the $n=4$ modes. By comparing results of Fig. 8 and Table I with the experimental measurements, we conclude that the results of the even core-localized mode are most consistent with the experimental data. In particular, the even core-localized mode has the largest $\gamma_{\alpha} / \gamma_{d}$, and has a radial structure core-localized on the low field side in agreement with the reflectometer data of Fig. 3. In contrast, all other modes are calculated to be significantly stable. The global modes extend radially well beyond $r / a=0.5$ as opposed to the observed localization to $r / a<0.5$. The odd mode is localized on the high field side and has a much higher frequency compared to that of the experimental value.

Now we turn to the $n=2$ mode. Figure 9 shows the radial displacement of $n=2$ even core-localized TAE and the corresponding density fluctuation and perturbed density. It is clear that the calculated $n=2$ mode has a ballooning mode structure localized on the low field side. In contrast, the measured $n=2$ mode shows a strong anti-ballooning feature (see 

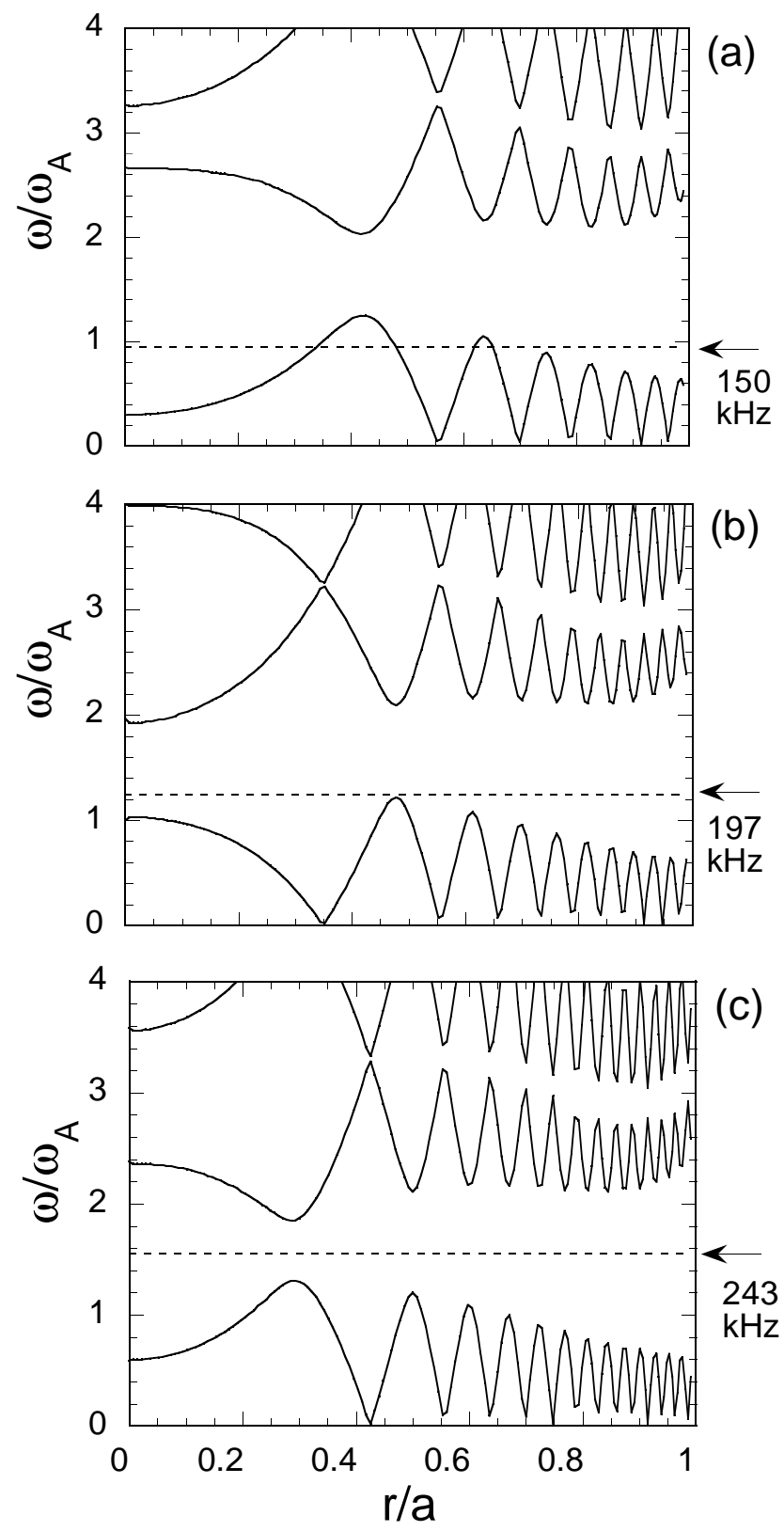

FIG. 5. The shear Alfvén continuum frequency $\omega / \omega_{A}$ versus normalized minor radius $r / a$ for (a) $n=2$, (b) $n=3$ and (c) $n=4$. The measured frequencies are indicated by the dotted lines. 


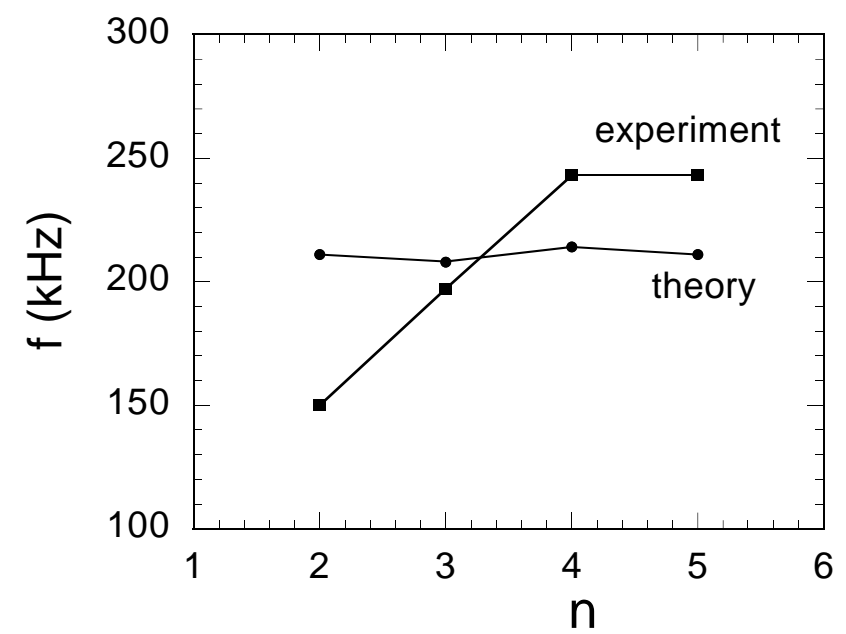

FIG. 6. Comparison between the calculated TAE frequency and the measured one for $n=2-5$ modes.

Fig. 3(b)). Similar to $n=4$, we have ruled out the $n=2$ odd mode because its calculated frequency is much higher than the measured value, although the expected anti-ballooning structure is consistent with the measurement.

From the above comparison of mode frequency, mode structure and mode stability, we can tentatively conclude that the observed $n=4$ mode is probably a TAE, and the $n=2$ is not. Our analysis for $n=3$ shows it is also a TAE mode.

We now compare the mode localization and stability in more detail. The stability result of the $n=4$ even core TAE is obtained for $q(0)=1.55$ which corresponds to a radial localization at $r / a=0.3$. This mode location is determined by the radius of the gap at $q=13 / 8=1.625$. As $q(0)$ changes, the mode location also changes due to variation in the gap location. Experimentally, there is some uncertainty in the measured value of $q(0)$ (or the central $q$ profile in general) and a typical error bar in $q(0)$ is about $10 \%$. Thus, the range of $q(0)$ is $q(0)=1.59 \pm 0.16$. Assuming the profile shape is fixed, the mode localization ranges from $r / a \sim 0$ to $r / a \sim 0.5$. For results of Fig. $7, q(0)$ is assumed to be $q(0)=1.55$ so that the $n=4$ mode location can better match the measured one. It is instructive to ask what is the expected mode location from stability consideration. In another words, how sensitive is the mode stability to the mode location or $q(0)$ ? Figure 10 shows the calculated ratio of alpha drive and total damping rate versus the mode location 

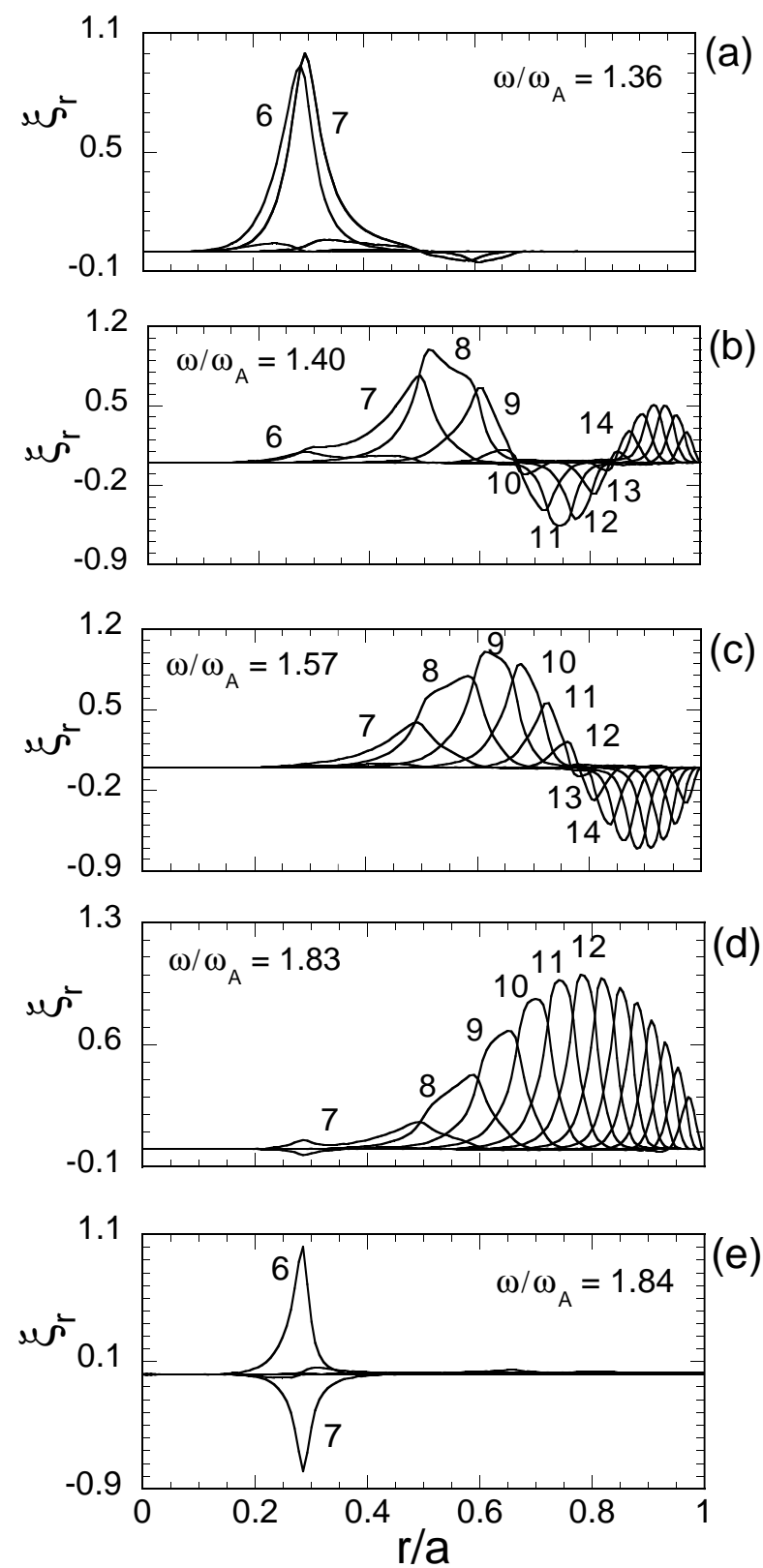

FIG. 7. The perturbed plasma displacement versus radius for five $n=4$ TAE modes. The mode frequency and poloidal harmonics are indicated in text. 

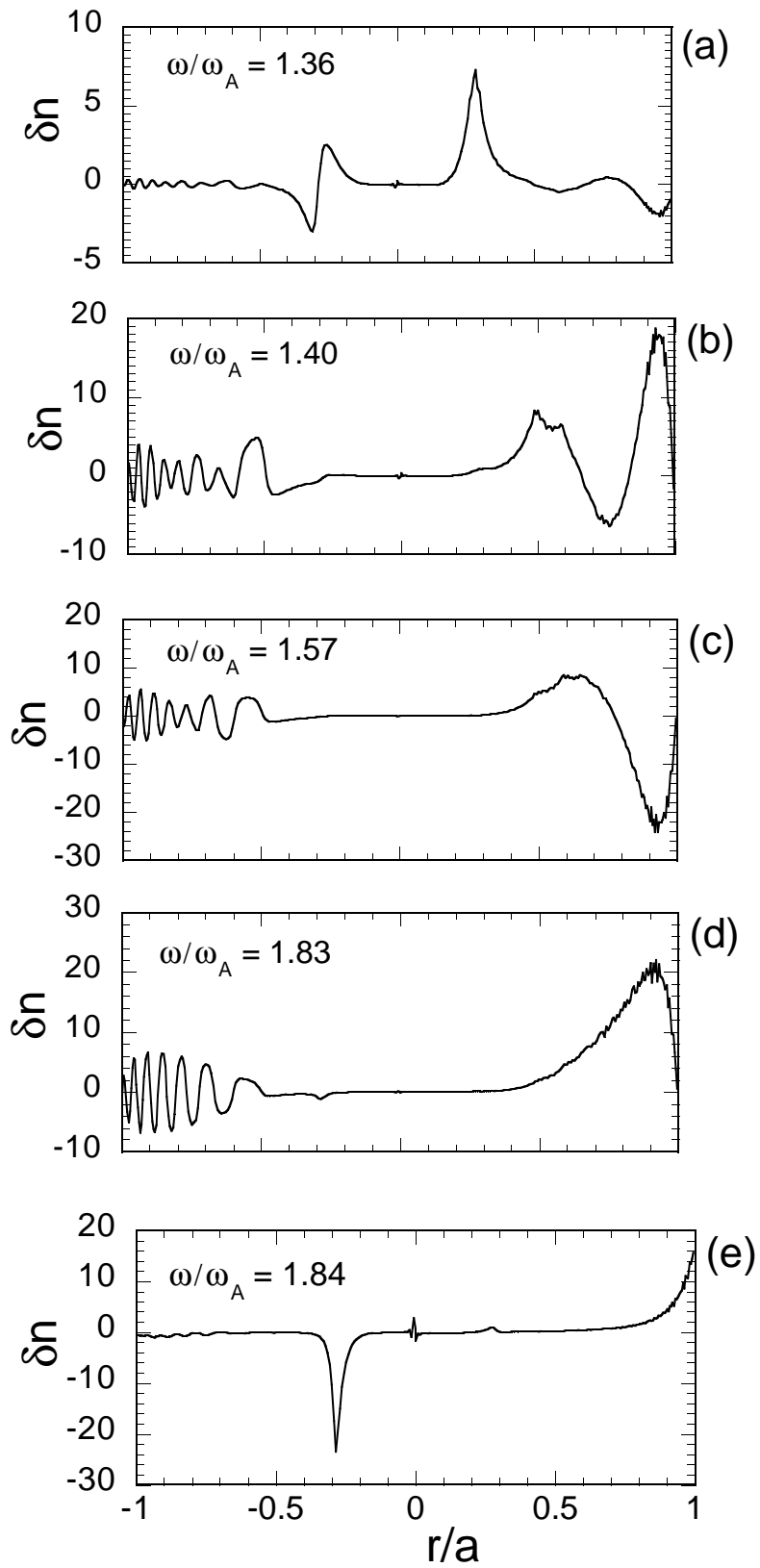

FIG. 8. The perturbed plasma densities correspond to the five $n=4$ TAE modes shown in Fig. 7 

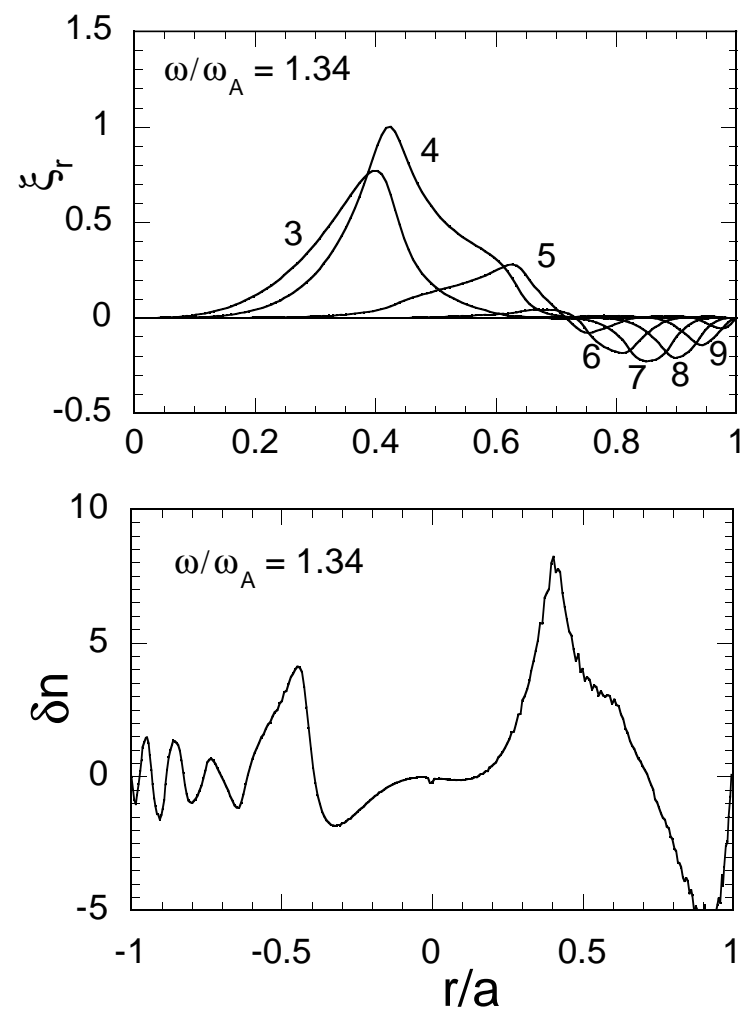

(b)

FIG. 9. (a) The perturbed plasma displacement and (b) the corresponding perturbed density for the $n=2$ even TAE mode.

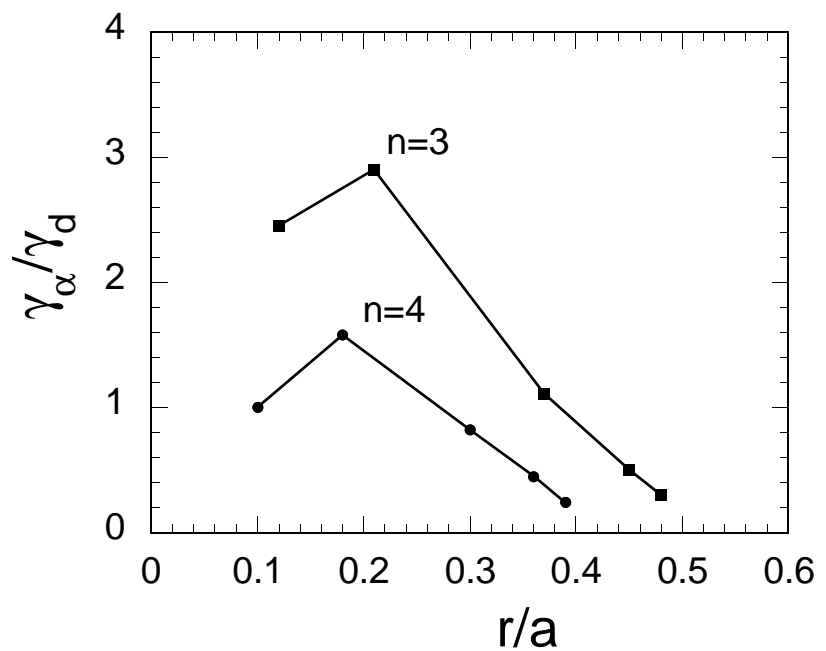

FIG. 10. The ratio of alpha drive to the total damping as a function of mode location $r / a$ for $n=3$ and $n=4$ even core-localized modes. 
for $n=4$ and $n=3$ even core-localized modes. The results are obtained by scanning $q(0)$ around the measured value of 1.59 while keeping fixed the value of $q^{\prime}(0), q(1)$, and $q^{\prime}(1)$. We see that the instability is maximized at about $r / a=0.2$, and the modes are unstable in the core region of $0.1<r / a<0.3$. On the other hand, all the global modes (which generally peak between half minor radius and the edge) are calculated to be stable. These results are consistent with the reflectometer's observation of mode location.

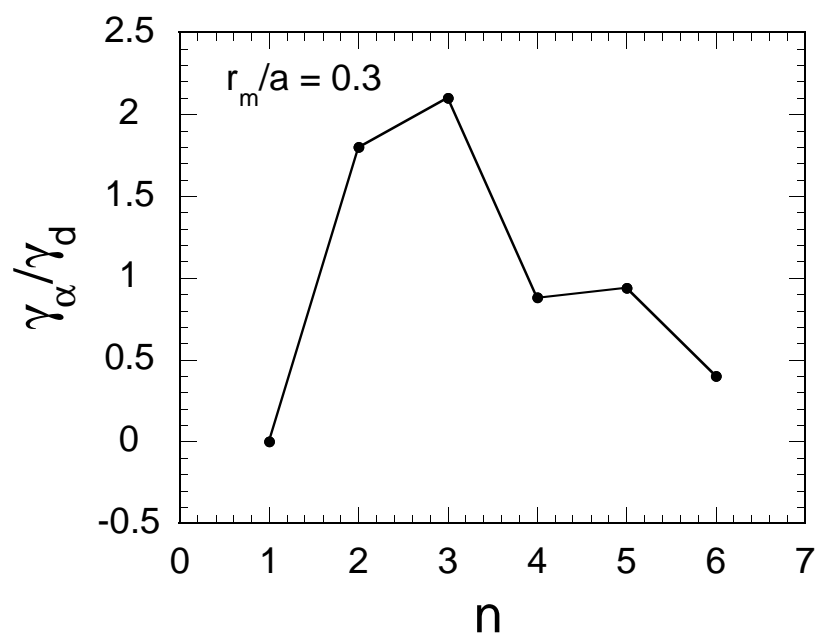

FIG. 11. Ratio of alpha drive to the total damping versus toroidal mode number $n$.

We now compare the mode spectrum of unstable TAEs. Figure 11 show the ratio of alpha drive to the total damping versus toroidal mode number assuming the modes are located at $r / a=0.3$ as observed in the experiments. Thus, for each $n$, a value of $q(0)$ near 1.59 is chosen to fix the mode location at $r / a=0.3$. The results show that the unstable mode number ranges from $n=2$ to $n=5$ approximately, again in agreement with the observations.

Finally, we consider the mode timing. Figure 12(a) shows the ratio of alpha drive to the total damping versus time for the $n=4$ even core-localized TAE and Fig. 12(b) shows separately the evolution of alpha drive $\gamma_{\alpha}$ (solid dots), the radiative damping $\gamma_{\mathrm{rad}}$ (solit triangles), the electron Landau damping (solid squares) and the ion Landau damping (circles). We see that the ratio $\gamma_{\alpha} / \gamma_{d}$ peaks at about $\mathrm{t}=2.9 \mathrm{sec}, 120 \mathrm{~ms}$ after the end of NBI. This is consistent with the observations of TAE activity which appears $100-150 \mathrm{~ms}$ 

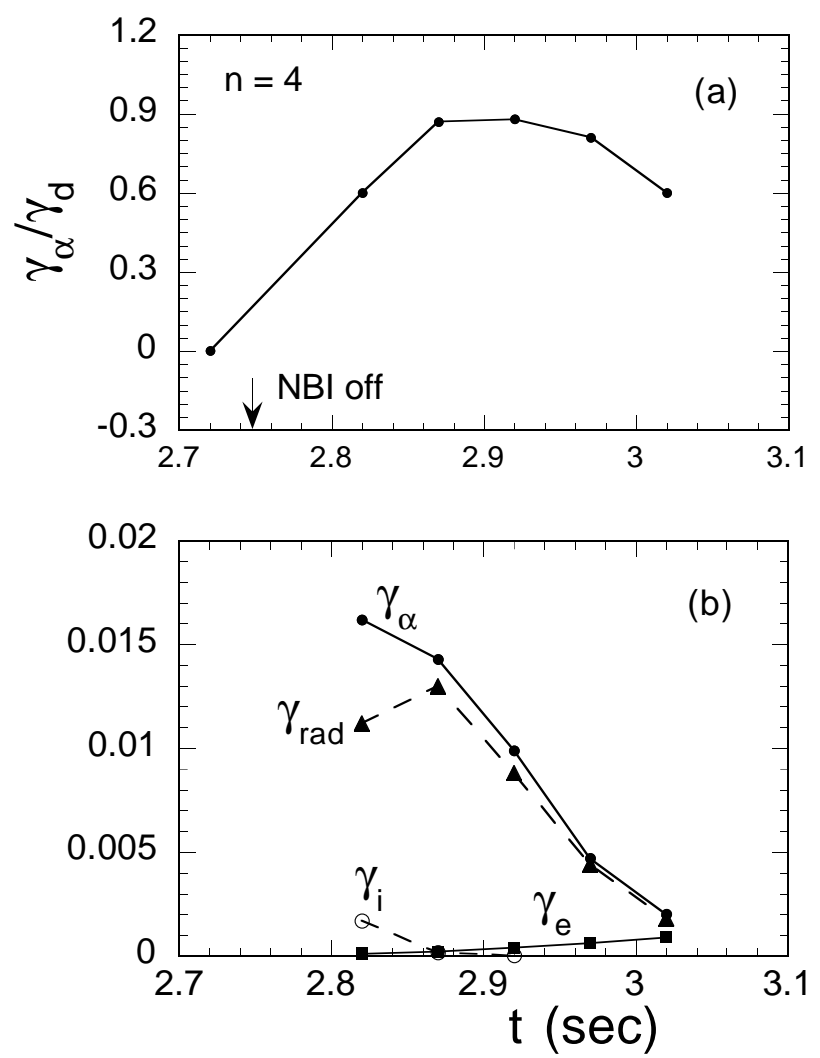

FIG. 12. (a) Evolution of ratio of alpha drive to the total damping; (b) Evolution of alpha drive $\gamma_{\alpha}$ (solid dots), the radiative damping $\gamma_{\text {rad }}$ (solit triangles), the electron Landau damping (solid squares) and the ion Landau damping (circles).

after the end of NBI. Physically, the core-localized modes do not exist in the continuum gaps during the NBI because the plasma pressure gradient is too large. Thus, $\gamma_{\alpha} / \gamma_{d} \approx 0$ at $t=2.72$. After the NBI is turned off, the plasma pressure decays on a time scale of $\approx 100 \mathrm{~ms}$ and falls below the critical value so that the core-localized TAEs can exist. The ratio $\gamma_{\alpha} / \gamma_{d}$ peaks around $120 \mathrm{~ms}$ after NBI due to the competition between rapidly changing drive and damping terms. After the end of NBI, both the alpha particle drive and total damping decreases in general. The alpha drive decreases because alpha particles slow down due to collision with electrons. Radiative damping, which is the dominant damping mechanism, also decreases in time because the ion and electron temperatures cool down. On the other hand, electron Landau damping increases in time. The ratio of drive to damping increases at first because the alpha drive decreases much slower than the damping. However, 
later on, the alpha drive decrease faster than the damping because of significant slowingdown in alpha particle velocity. The results of Fig. 12 are obtained for fixed q profile at $q(0)=1.55$. In the actual experiment, the $q$ profile evolves in time which should contribute significantly to the evolution of mode stability as suggested by results of Fig. 10 . The $q$ profile evolution could also lead to sequential excitation of muti- $n$ modes as observed. However, a precise comparison with the experimental observations is complicated by the experimental uncertainty in the $q$ profile $(\approx 10 \%)$ which is still sufficiently large to obscure subtle changes related to the mode evolution. Thus, $q$ profile is assumed to be fixed for simplicity.

\section{DISCUSSIONS AND CONCLUSIONS}

We have carried out comprehensive study of alpha-driven TAE in the TFTR DT plasmas with weak central shear and $q(0)>1$. In most cases, the observed alpha-driven Alfvén frequency activity is consistent with the expected ballooning even core-localized TAE modes for $n>2$. Several key observations can be explained quantitatively by our theory. First, the modes are core-localized to within $r / a=0.4$. Second, the modes can only be excited by alpha particles after the termination of NBI. Third, the mode frequencies lie inside the continuum gaps (for $n>2$ ). Finally, the calculated stability threshold in alpha particle beta agrees with the measurements within a factor of two. However, significant discrepancies remain. In particular, the observed $n=2$ mode appears to be "anomalous" and has many features not understood by the present theory. Among them, the mode is observed to have strongly anti-ballooning structure and the frequency inside the calculated Alfvén continuum at the observed mode radial location.

These discrepancies might be resolved individually, but not consistently as a whole. For example, the odd $n=2$ mode can explain the anti-ballooning mode structure, but it has a much higher frequency than the experimental value ( by a factor of two). Also, the frequency of the even $n=2$ mode could match the experimental value by adjusting $q(0)$ and plasma 
density within their uncertainties, but the calculated mode is localized on low field side, which is opposite to the observed anti-ballooning feature. It should also be pointed out that the calculated mode frequency dependence on mode number is much too weak as compared to the observed one (see Fig. 5).

There are several possible candidates for the $n=2$ mode. One is the Energetic Particle Mode or EPM which is intrinsically driven by energetic particles. The EPM's frequency is generally inside the Alfvén continuum and thus can explain the low frequency observed. However, this mode usually requires a large drive to overcome the continuum damping. The alpha particle beta in the experiments seems to be too small to make EPM a viable candidate. Another possibility is the kinetic Alfvén wave (KAW) which localized at the minimum of the $m=3$ shear Alfvén continuum. This requires a reversed $q$ profile with $q_{\text {min }} \approx 1.7$ at $r / a=0.3$ which still lies within the experimental uncertainty of the MSE measurement. However, the KAW is also unlikely because it is expected to have a homogeneous poloidal mode structure (neither ballooning or anti-ballooning), which is not consistent with the observed mode structure.

In summary, the detailed experimental observations of alpha-driven TAE in TFTR puts a stringent test to the theory. Although the present model agrees reasonably well with the measurements in mode frequency, mode localization and mode stability for $n>2$ modes, significant discrepancy remains for the $n=2$ mode. It is a challenge both for theory and experiment to understand this anomalous mode.

The authors gratefully acknowledge the support of the TFTR group. We also thank Prof. H. Berk for reading the manuscript and many useful discussions. This work is supported by the U.S. Department of Energy under Contract No. DE-AC02-76-CHO-3073. 


\section{REFERENCES}

${ }^{1}$ G. Y. Fu and J. W. Van Dam, Phys. Fluids B1, 1949 (1989).

${ }^{2}$ K. L. Wong, R. J. Fonck, S. F. Paul et al., Phys. Rev. Lett. 66, 1874 (1991).

${ }^{3}$ W. W. Heidbrink, E. J. Strait, E. Doyle, G. Sager, and R. Snider, Nucl. Fusion 31, 1635 (1991).

${ }^{4}$ J. R. Wilson, M. G. Bell, H. Biglari et al., in Proceedings of the Fourteen International Conference on Plasma Physics and Controlled Nuclear Fusion Research, Wurzburg, Germany, September, 1992 (International Atomic Energy Agency), IAEA-CN-56/E-2-2.

${ }^{5}$ E. Fredrickson, S. Batha, M. Bell et al., in Proceedings of the Fifteen International Conference on Plasma Physics and Controlled Nuclear Fusion Research, Seville, Spain, October, 1994 (International Atomic Energy Agency), paper IAEA-CN/60/A-2-II-5.

${ }^{6}$ M. Saigusa, H. Kimura, S. Moriyama et al., Plasma Phys. Control. Fusion 37, 295 (1995).

${ }^{7}$ H. Kimura, M. Saigusa, S. Moriyama et al., Phys. Lett. A 199, 86 (1995).

${ }^{8}$ S. Ali-Arshad and D. J. Campbell, Plasma Phys. Control. Fusion 37, 715 (1995).

${ }^{9}$ A. Fasoli, R. Heeter, D. Borba, C. Gormezano, S. Sharapov, and A. Juan, Bull. Am. Phys. Soc. 42, 1860 (1997).

${ }^{10}$ K. M. Young, M. G. Bell, W. R. Blanchard et al., Plasma Phys. Controlled Fusion 26, 11 (1984).

${ }^{11}$ R. Nazikian, G. Y. Fu, S. H. Batha et al., Phys. Rev. lett. 78, 2976 (1997)

${ }^{12}$ Z. Chang, R. Nazikian, G. Y. Fu et al., Phys. Plasmas 4, 1610 (1997).

${ }^{13}$ R. V. Budny, M. G. Bell, H. Biglari et al., Nucl. Fusion 32, 429 (1992).

${ }^{14}$ G. Y. Fu, C. Z. Cheng, R. Budny et al., Phys. Plasmas 3, 4036 (1996)

${ }^{15}$ R. Nazikian, G. Y. Fu, Z. Chang et al., Phys. Plasmas (1998). 
${ }^{16}$ D. A. Spong, B. A. Carreras, C. L. Hedrick, J.-N. Leboeuf, A. Weller, in Proceedings of the Fifteen International Conference on Plasma Physics and Controlled Nuclear Fusion Research, Seville, Spain, October, 1994 (International Atomic Energy Agency), paper IAEA-CN-60/D-P-II-3.

${ }^{17}$ C. Z. Cheng, L. Chen, and M. S. Chance, Ann. Phys. (N. Y.) 161, 21 (1985); C. Z. Cheng and M. S. Chance, Phys. Fluids 29,3695 (1986).

${ }^{18}$ C. E. Kieras and J. A. Tataronis, J. Plasma Phys. 28, 395 (1984).

${ }^{19}$ G. Y. Fu and C. Z. Cheng, Phys. Fluids B2, 985 (1990).

${ }^{20}$ G. Y. Fu, C. Z. Cheng, R. Budny, Z. Chang, D. S. Darrow, E. Fredrickson, E. Mazzucato, R. Nazikian, and S. Zweben, Phys. Rev. Lett. 75, 2336 (1995).

${ }^{21}$ G. Y. Fu, Phys. Plasmas 2, 1029 (1995).

${ }^{22}$ H. L. Berk, J. W. Van Dam, D. Borba, J. Candy, G. T. Huysmans, and S. Sharapov, Phys. Plasmas 2, 3401 (1995).

${ }^{23}$ J. Candy, B. Breizman, J. W. Van Dam, and T. Ozeki, Phys. Lett. A 215, 299 (1996).

${ }^{24}$ R. Mett and S. Mahajan, Phys. Fluids B 4, 2885 (1992).

${ }^{25}$ W. W. Heidbrink, E. J. Strait, M. S. Chu, A. D. Turnbull, Phys. Rev. Lett. 71, 855 (1993).

${ }^{26}$ R. Betti and J.P. Freidberg, Phys. Fluids B3, 1865 (1991)

${ }^{27}$ K. Appert, R. Gruber, F. Troyon, and J. Vaclavik, Plasma Phys. 24, 1147 (1982)

${ }^{28}$ D.W. Ross, G.L. Chen, and S.M. Mahajan, Phys. Fluids 25, 652 (1982)

${ }^{29}$ L. Chen and A. Hasegawa, Phys. Fluids 17, 1399 (1974).

${ }^{30}$ L. Chen, Phys. Plasmas 1, 1519 (1994)

${ }^{31}$ C. Z. Cheng, N. N. Gorelenkov, C. T. Hsu, Nucl. Fusion 35, 1639 (1995). 
${ }^{32}$ M. N. Rosenbluth and P. H. Rutherford, Phys. Rev. Lett. 34, 1428 (1975).

${ }^{33}$ A. B. Mikhailovskii, Zh. Eksp. Teor. Fiz. 68, 1772 (1975) [Sov. Phys. JETP 41, 890 (1975)

${ }^{34}$ C. Z. Cheng, Phys. Reports 211, 1 (1992).

${ }^{35}$ G. Y. Fu, C. Z. Cheng and K. L. Wong, Phys. Fluids B5, 4040 (1993).

${ }^{36}$ R. V. Budny, Nucl. Fusion 34, 1247 (1994); R. V. Budny, M. G. Bell, A. C. Janos, D. L. Jassby, L. C. Johnson, D. K. Mansfield, D. C. McCune, M. H. Redi, J. F. Schivell, G. Taylor, T. B. Terpstra, M. C. Zarnstorff, S. J. Zweben, Nucl. Fusion 35, 1497 (1995). 
TABLE I. The calculated mode frequency, mode type, mode stability of the $n=4$ TAE modes.

\begin{tabular}{ccccc}
\hline \hline mode & type & $\omega / \omega_{A}$ & $f(\mathrm{kHz})$ & $\gamma_{\alpha} / \gamma_{d}$ \\
\hline $\mathrm{a}$ & even core-localized & 1.36 & 215 & 0.82 \\
$\mathrm{~b}$ & global & 1.40 & 220 & 0.17 \\
$\mathrm{c}$ & global & 1.57 & 246 & 0.18 \\
$\mathrm{~d}$ & global & 1.83 & 287 & 0.05 \\
$\mathrm{e}$ & odd core-localized & 1.84 & 289 & 0.63 \\
\hline \hline
\end{tabular}

